\title{
Models and theories of health behavior and clinical interventions in aging: a contemporary, integrative approach
}

This article was published in the following Dove Press journal:

Clinical Interventions in Aging

\section{W Jack Rejeski \\ Jason Fanning}

Department of Health \& Exercise Science, Wake Forest University, Winston-Salem, NC 27I09, USA
Correspondence: W Jack Rejeski Wake Forest University, Department of Health and Exercise Science, Box 7868,

Winston-Salem, NC 27109, USA

Tel + I 3367585837

Email rejeski@wfu.edu
Background: Historically, influential models and theories of health behavior employed in aging research view human behavior as determined by conscious processes that involve intentional motives and beliefs. We examine the evolution, strengths, and weaknesses of this approach; then offer a contemporary definition of the mind, provide support for it, and discuss the implications it has for the design of behavioral interventions in research on aging.

Methods: A narrative review was conducted.

Results: Traditionally, models and theories used to either predict or change health behaviors in aging have not viewed the mind as encompassing embodied and relational processes nor have they given adequate attention to multi-level, in-the-moment determinants of health behavior.

Discussion: Future theory and research in aging would benefit from a broader integrative model of health behavior. The effects of adverse life experience and changes in biological systems with aging and chronic disease on health behavior warrant increased attention.

Keywords: health behavior, models, theories, interventions, aging

\section{Introduction}

The health care of older adults is complex requiring varying degrees of commitment on the part of patients to follow prescribed regimens of treatment. These regimens include behaviors such as dietary intake, physical activity, prescription drug use, taking preventive health screenings, and adherence to behavior protocols for physical rehabilitation. As a field, Behavioral Medicine has come to recognize that health behaviors are determined by multiple levels of influence. ${ }^{1}$ For example, significant others and interactions with health care providers play a powerful role in shaping the beliefs of older adults. Similarly, what older adults would "like to do" and what they are "able to do" in the realm of health behavior is often determined, in part, by environmental and policy decisions such as access to facilities and reimbursement from Medicare. Of critical importance is that, while theories often conceptualize health behaviors as intentional and under conscious control, this is often not true as is evident in the biological and environmental determinants of addictive behaviors. ${ }^{2}$

We open this review by touching on several models and theories of health behavior and/or health behavior change, capturing evolving thought on the topic. Our goal is to demonstrate how models/theories of health behavior have evolved across time and gaps that exist. We then present a contemporary definition for the 
concept of mind and review support for an integrative model based on this perspective. We believe this model will help to advance intervention development in aging research and foster an interdisciplinary science of health behavior and health behavior change.

\section{A progression in models/theories of health behavior and behavior change}

Behavioral scientists have devoted considerable effort to the development and evaluation of models and theories designed to understand and/or influence health behavior. As theory has advanced, scientists have adopted increasing specificity in the conceptual definition and measurement of constructs while becoming more interested in behavior change over understanding why individuals engage in particular health behaviors. Additionally, there has been increased interest in affect as well as the physiological and environmental input to health behavior and health behavior change. To illustrate the evolution of extant models/theories and the current state-ofthe-art, we discuss the health belief model, the Social Cognitive Theory, the relapse prevention model, selfdetermination theory, research on affect and a biological model of desire, along with the socio-ecological model.

\section{Health belief model (HBM)}

The HBM first appeared in the 1950s as a guide to research on tuberculosis screening. ${ }^{3,4}$ It distilled concepts from an established body of psychological and behavioral research and set the stage for the theories that followed. HBM is an expectancy-value model. As an example, people take medication to control their cholesterol because they value avoiding cardiovascular disease. Core constructs include perceived threat of a given disease state, which is the product of perceived susceptibility to the disease and perceived disease severity. The model also emphasizes decisional balance: the relative weight of perceived benefits as compared to perceived barriers to engaging in a behavior. As shown in Figure 1, health behavior results from the combined effect of perceived threat and decisional balance over anticipated outcomes. ${ }^{4}$ The HBM acknowledges the input on health behavior from other factors such as psychosocial variables and environmental cues, but it conceptualizes these effects as acting through either perceived threat or decisional balance. Of note, HBM practitioners have long recognized the limited scope of the model. For instance, as Janz and Becker noted: " ${ }^{4}$ "It is clear that other forces influence health actions as well; for example...some behaviors (eg, cigarette smoking; tooth-brushing) have a strong habitual component obviating any ongoing psychosocial decision-making process".

\section{Social cognitive theory (SCT)}

As a second approach to models/theories of health behavior, we focus on Bandura's SCT. ${ }^{5}$ As with HBM, SCT conceptualizes individuals as rational actors. While there is continued emphasis on the concept of expectancy-value, a chief advancement of SCT is its focus on personal agency and the importance of context as a determinant of health behavior. Moreover, while SCT has been useful in understanding why people perform a specific health behavior, it has also had a major effect on interventions for behavior change.

Self-efficacy, or one's perceived ability to bring about a specific course of action in a particular context, is the core construct in SCT. Efficacy beliefs are dynamic, affecting and being affected by several downstream constructs highlighted in SCT (see Figure 2). These include outcome expectations and barriers/facilitators of behavior that arise from both social relations and cultural forces. Individuals with higher self-efficacy for a behavior are likely to have higher expectations for associated outcomes. They also perceive greater support from the social and

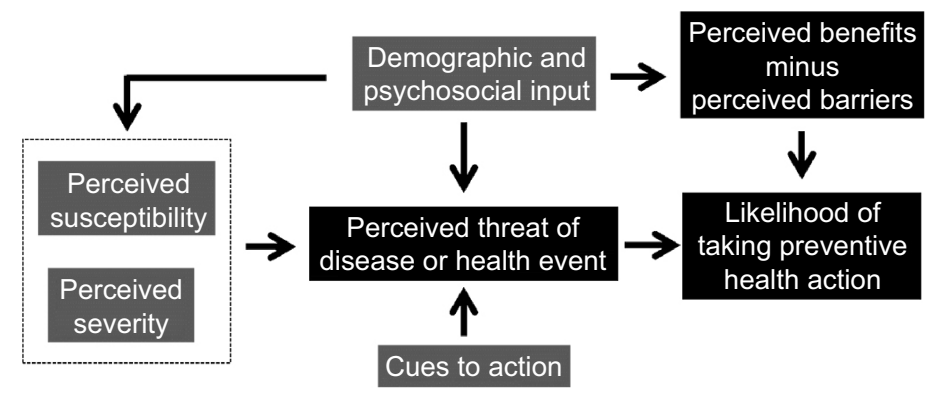

Figure I The health belief model.

Note: Adapted from Janz NK, Becker MH. The health belief model - a decade later. Health Ed Quart. 1984;II(I):I-47. Copyright I984, with permission from SAGE Publications. ${ }^{4}$ 


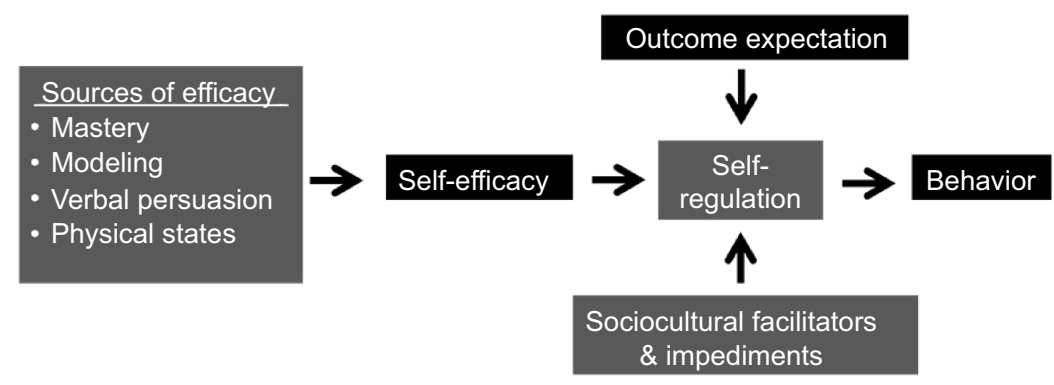

Figure 2 Social cognitive theory.

Note: Aadapted from Bandura A. Health promotion by social cognitive means. Health Educ Behav. 2004;3I(2): 143-164, copyrught 2004 by permission of SAGE Publications. ${ }^{6}$

physical environment and engage in more favorable selfregulatory behaviors than those with low self-efficacy. Success with the behavior fuels self-efficacy, especially when success occurs in the face of challenge. In addition, encouragement from others and observing relatable peers or those less skilled having success with a given behavior also enhances self-efficacy. Finally, one's physiological state has an immediate influence on self-efficacy. For example, Bandura calls forth the image of preparing for a public speaking event. As anxiety mounts in preparing to deliver a talk, some individuals become hypersensitive to physical symptoms such as rising heart rate, increasingly sweaty palms, and a queasy stomach. The result is that they experience a sharp, in-the-moment decline in their speech-related self-efficacy.

In part, the appeal of SCT arises from its specificity. ${ }^{7}$ Other contemporary theories, such as the theory of planned behavior, prioritize parsimony and do not address behavior change. ${ }^{8,9}$ SCT also offers interventionists clear targets for improving efficacy beliefs, supporting self-regulation, minimizing external barriers, and bolstering positive outcome expectancies. Moreover, it explicitly models the interplay between underlying transient biological states, one's sense of agency, and the influence of proximal socio-structural pressure. Unfortunately, these key considerations are typically lost in implementation, with the focus constrained to individual-level perceptions and the influence of proximal social connections. ${ }^{7}$

\section{Relapse prevention (RP)}

The third model of health behavior that we chose for inclusion is RP for addictive behavior. ${ }^{10} \mathrm{RP}$ is a model targeted specifically to behavior change. As an outgrowth of SCT, the intent behind RP was to describe the process of relapse for addictive behavior, emphasizing the importance of early intervention. They conceptualized relapse as an expected and transitional process; a key aim is to avoid or to learn how to cope with high-risk situations.
RP identified two categories of factors that contribute to a risk for relapse: immediate determinants and covert antecedents. Akin to Bandura's recognition that transient, in-the -moment physiological states can exert substantial influence on self-efficacy, RP proposes that high-risk situations serve a similar function. They are immediate (in-the-moment) determinants of addictive behavior. These range from social and physical environments, to internal states such as depression or negative affect. Another immediate determinant, coping, captures how an individual responds to a high-risk situation. Outcome expectancies are a third determinant, in that individuals who expect short-term benefits such as reduced anxiety from the behavior are more likely to relapse. The fourth immediate determinant is the abstinence violation effect, which refers to the feeling of guilt or lack of control accompanying a single lapse.

Covert antecedents of relapse are a partial determinant of whether an individual successfully negotiates immediate determinants. Here, lifestyle factors, including both positive and pleasurable activities alongside one's responsibilities contribute to or alleviate stress, which in turn is related to the likelihood of a relapse. More recent iterations of the model $^{11}$ specify both trait-like-tonic-influences on relapse, which are thought to dictate initial susceptibility to a relapse, and more dynamic and transient influences - phasic. Phasic influences include momentary mood states, urges and cravings, and in-the-moment changes in self-efficacy or outcome expectations. These phasic influences represent the most proximal determinants of a relapse.

Although not explicitly stated in RP, an interesting feature is the awareness that conscious goals related to recovery often succumb to the physiological symptoms of withdrawal, negative affective states, and the emotional tipping point created by the abstinence violation effect. Thus, it is not surprising to find that recent research on mindfulness-based treatment techniques specific to RP (MBRP) have been successful in countering the influence of negative affective states 
on the likelihood of relapse, and enhancing individuals' abilities to cope with distress. ${ }^{12,13}$

\section{Self-determination theory (SDT)}

We believe it is important to briefly discuss Deci and Ryan's $\mathrm{SDT}^{14}$ because it unites concepts from SCT (eg, goal setting; mastery), RP (eg, one's inner state affects motivated behavior), the motivational role of affect in behavior by way of enjoyment, and the importance of strong social ties. SDT posits that humans are driven by three core needs: the need to experience competence, meaningful social connection (ie, relatedness), and autonomy (ie, a sense of control over one's behaviors). The core needs outlined in SDT are positioned to be innately valued, and as with other theories, Deci and Ryan underscore the importance of aligning the content of one's goals with an individual's core needs. ${ }^{14}$ For instance, an exercise goal formed for the explicit purpose of looking better to one's peers, an extrinsic personal goal, will lose salience more rapidly than an intrinsic exercise goal emanating from the value of human connection and formed for the purpose of being able to engage with one's grandchildren or to foster a relationship with friends. ${ }^{15}$

Moreover, the ways in which these goal-driven behaviors are regulated are given importance in SDT. An intrinsically motivated behavior is one that brings about feelings of interest, enjoyment, or satisfaction, and it is theorized that this produces self-motivated, or selfdetermined behavior that is likely to last. When the behavior is motivated by factors aside from the merits of the behavior itself, it is said to be externally regulated. These more "controlling" forms of motivation are expected to sometimes regulate short-term behavior, but have a low likelihood of facilitating behavioral maintenance. ${ }^{15}$

There are several important conclusions to be drawn from research on SDT and health behavior. As with research on incentives and affective valence described below, SDT highlights the importance of maximizing behaviors that produce positive bodily states such as enjoyment. It also provides a useful framework for considering appropriate incentives. Namely, by emphasizing incentives that are intrinsic as opposed to extrinsic. Lastly, it underscores the value of leveraging the group as a tool of behavior change; a notion we will highlight in the final section of this manuscript.

\section{Incentives/affect}

Although the motivational significance of incentives and affective valence that people associate with particular outcomes of a health behavior are evident in the concept of expectancy-value, within contemporary theoretical frameworks it is frequently assumed that people value their health and the focus of most research has been on selfefficacy, outcomes expectation, and behavioral intention. ${ }^{7}$ Researchers traditionally assumed that increases in selfefficacy are valued because they increase a sense of personal agency. ${ }^{5,16}$ One exception is research on RP in which researchers clearly appreciate the role of physiological withdrawal on relapse and the fact that addictive substances are often valued as a means of coping with life stress. ${ }^{11}$

There has been a surge of interest in the affective determinants of health behavior, including work on both reflective and reflexive affect. ${ }^{17}$ Reflective affect is cognitive based and referred to as "subjective liking", whereas reflexive affect has been characterized as "core liking", the pure, abrupt, visceral experience that is a function of contextual stimuli and associations. ${ }^{18}$ Reflexive affect can be an in-the-moment experience or anticipatory in nature. Rhodes and Gray ${ }^{19}$ recently note that most research on affect and health behavior has focused on reflective as opposed to reflexive affect. Although not conducted on older adults, reviews of the exercise literature have shown that reflexive affect may be more important in predicting future exercise behavior than reflective affect or social cognitive variables. ${ }^{20}$

Given the growing interest in reflexive affect ${ }^{17}$ and the importance of incentives to health behavior, there are important lessons to be learned from work in the biology of addiction. In the "Biology of Desire", Lewis ${ }^{2}$ describes the neuroscience of how substances and behaviors of desire become habitual through activity in the reward network. The central axis for desire begins in the ventral tegmental area (VTA) of the midbrain. Activation of this region of interest provides the fuel for desire-dopamine! Other key areas of the brain involved in impulsive behavior - the initiation of an addictive behavior-include the ventral and dorsal striatum, amygdala, hippocampus, and prefrontal cortex (PFC). In the early stages of desire for a substance or behavior, both nonconscious and conscious processing are involved. The amygdala acquires and maintains emotional sensations and communicates with the hippocampus, a structure that stores explicit memories of experience. The ventral striatum is responsible for feelings of attraction, desire, and craving. It is the main driver for impulsivity, getting its fuel from the VTA. The PFC creates conscious, context-specific interpretations of highly motivating situations and is key to executive function, 
planning, bringing memories into consciousness, sorting and comparing memories, and making decisions.

Once a person has been repeatedly exposed to a desired substance or behavior, involvement of the PFC in the reward network weakens to the point where conscious processing is no longer involved - the dorsal lateral region of the striatum has led to addiction, a compulsive act. The substance or behavior is now a habit: stimuli lead to a response (S-R) in the absence of conscious thought. We believe this model describing the biology of desire is important for several reasons. First, desire - or the incentive value of a behavior - is applicable to both functional and dysfunctional health behaviors. Second, as this model illustrates, intervention development would benefit from integrating concepts from neuroscience into the study of health behavior change. Third, as we will see later, there may be important neural phenotypes that could assist in tailoring treatment. Fourth, we believe this model is applicable to understanding incentives or desire more generally; habits vary in their strength! If we hope to promote health behaviors among older adults, there is little question that we need to discover the motivational levers that operate for different people in varied contexts. Fifth, we believe a focus on desire has wide application to the design of behavioral interventions and should give pause to health scientists implementing aversive interventions such as highly popular high-intensity physical activity training regimens.

\section{Socio-ecological models}

Finally, it is important to note the growing popularity of ecological models of health behavior. Drawn from a biological sciences view of ecology, which is interested in capturing the interplay between an organism and its environment, socio-ecological models identify multiple levels of influence, typically ranging from individual factors such as one's biological state to broader community, geopolitical, and policy influences. ${ }^{21-23}$

A key assumption of these models is that researchers can study individuals at various levels of influence, including the individual, community, state, or national level. However, effective health behavior change likely needs to consider the individual as affected by these various levels of influence. For instance, the likelihood an individual sets a goal to eat better, engage in exercise, commute in an active manner, or reduce sitting will be influenced by their built (eg, are there bike paths and healthy food options?) and social (eg, do social norms support healthy behavior?) environments. Similarly, the extent to which the environment is low-stress and perceived as safe may help or hinder an individuals' ability to adhere to behavioral goals. ${ }^{24,25}$ They also recognize that environments and those existing within them are in a constant state of flux; thus, interventions should be flexible and adaptable. ${ }^{23}$ Clearly, social-ecological approaches to behavior change require considerable resources and time relative to individual-level interventions; however, they also underscore the important role that social and physical environments have on health behaviors, a point we will come back to later.

\section{Summary}

Across the models/theories reviewed, there is general acceptance for the concept of expectancy-value. That is, people engage in health behaviors because of the belief that the behavior will yield outcomes of value. It is interesting to note that, with the emergence of SCT, the focus has been on self-efficacy even though it is one of the several core constructs alongside incentives and outcome expectations. Although the role of affect and physiological states on health behavior is apparent in SCT, the theory posits that self-efficacy mediates these effects. In addition, it is surprising that researchers have paid so little attention to the incentives underlying health behaviors, how incentives and goals benefit from being linked to core needs central to SCT, and how the affect associated with the incentive value of health behavior may be tempered by the sacrifices that older adults are often required to make when changing their behavior.

Of note is the fact that, as models and theories of health behavior have evolved, there has been an increasing conceptual focus on behavior change. In fact, RP identified the importance of phasic determinants of behavioral maintenance, emphasizing the role of reflexive affect. Peoples' psychological and physiological states can change over relatively brief periods and cause dramatic shifts in behavioral intentions. Finally, as far back as 1984, Janz and Becker recognized that conscious, decision-based models such as HBM could not explain all health behaviors, specifically noting the habitual drive underlying behaviors such as cigarette smoking. Supported by recent trends in neuropsychology, future research in intervention development must consider the role played by nonconscious processes and, in particular, how to modify these processes.

\section{The concept of mind: theory development and scientific inquiry}

We believe there is merit in stepping back for a moment to reconsider the concept of "mind" in greater depth. The reason 
for this reflection is that how theoreticians/researchers think about the mind heavily influences what they believe to be the primary drivers of behavior. Traditionally separated from the body, behavioral science has conventionally viewed the mind as a faculty of being human that enables people to have an awareness of the world and of their experience; it is responsible for consciousness and gives humans the capacity to think and to feel. The role of the mind or lack thereof in theory development is perhaps most evident the classic work of B.F. Skinner. Skinner proposed that the mind was irrelevant to understanding human behavior; rather, he argued that people behave in response to operant conditioning to reinforcement and/or punishment; promoting the concept of environmental engineering as a means for shaping behavior. Even in contemporary thinking, concepts such as "nudging", 26 popular in behavioral economics, have shown that some desired health behaviors can be achieved through positive incentives or indirect influence; reemphasizing the point that in some instances the mind, when defined by traditional criteria of awareness, thinking, and feeling, is irrelevant to human behavior. Alternatively, the cognitive revolution that followed Behaviorism and continues to be favored by many theoreticians, places an emphasis on conscious, cognitive processes as determinants of health behavior. $^{7}$

As we consider why older adults do or do not behave optimally within the context of medical research or health care, we will continue to reinforce the notion that the health behavior of older adults requires considering multiple levels of influence, some of which obviate the need for conscious decision-making. We will also emphasize that human behavior is not always rational, and that implicit memories and biased processing of information are more common than currently recognized. Most important, we believe that a more complete understanding of why older adults behave as they do within the context of medical research and health care would emerge from a broader, alternative view of the mind. Specifically, we adopt the position that the mind should be conceptualized as a process rather than as an outcome such as a thought or feeling, noting that this process is responsible for regulating energy and information flow, and that this process is both embodied and relational.

\section{The Mind as a Process and Implications for Health Behavior}

Paraphrasing Siegel, ${ }^{27}$ the human mind is a process that regulates the flow of energy and information between the body, brain, and relationships - thus, it is both embodied

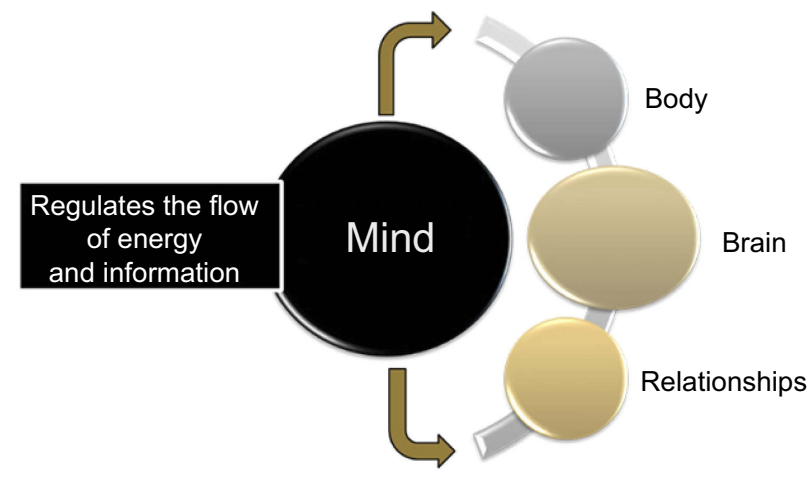

Figure 3 The mind as a process.

Note: Reprinted from Lucas AR, Klepin HD, Porges SW, Rejeski WJ. Mindfulnessbased movement: a polyvagal perspective. Integrative Cancer Therapies. 20I8; I7(I):5$15^{30}$

and relational (see Figure 3). As we will soon demonstrate, defining the mind as a process is consistent with Hebb's ${ }^{28}$ concept of associative learning: neurons that fire together wire together. What begins as energy through activation of neurons eventually becomes information that then defines learning and the formation of memories. Furthermore, as Siegel pointed out, the flow of energy and information occurs not only in the brain, but in conjunction with the body and relationships as well. Conceptualizing the mind as embodied is critically important to understanding health behavior for two reasons. First, it positions various biological inputs that may be either stable or unstable as important determinants of subjective experience and behavior. And second, Glass and McAtee ${ }^{29}$ argue that features of the social, built and natural environment become embodied and act as "risk-regulators" that effect health behavior via various biological pathways. In other words, toxic environments adversely affect biological regulatory systems. These systems then become "internal risk regulators" that can have powerful effects on health behavior.

This complex, co-dependency between molecules, the mind, and the environment has also been supported by McEwen $^{31}$ and is obvious in the area of drug addiction where toxic microenvironments influence exposure to drugs $^{32}$ that then lead to molecular and cellular adaptations in the body that result in drug abuse. ${ }^{33}$ Drug abuse also leads to other behaviors that can compromise health such as exposure to violence and a rapid drop off in self-care.

When Siegel noted that the mind is relational, he emphasized that the human brain is engaged in a constant flow of energy and information with other people. In fact, as we have just described, micro-social environments serve as a "risk regulator" of drug use. The powerful role of social 
relationships on health behavior is not surprising. We all enter this world dependent on others for our survival; as one leading neuroscientist puts it, our brains are wired to connect with others. ${ }^{34}$ It is important to note that Siegel's focus on the relational mind emphasized the effect that attachment through close interpersonal relations in childhood has on behavior and well-being. We agree that early interpersonal attachment experience plays an important role in health behavior not only in infancy but also across the lifespan. However, as we note above and consistent with Glass and McAtee, ${ }^{29}$ we would argue that the relational mind encompasses powerful influences from social, built, and natural environments that range from the micro to macro levels of analysis.

Figure 4 provides a conceptual model of health behavior that describes the interrelationships between the relational mind (box to the left) and the biological regulatory systems that embody relational experiences (the box to the right). Embodiment occurs when relational experiences alter biological regulatory systems (BRS) through their effects on genetic and biological substrates of these systems. Note the distinction between the body and brain in depicting the BRS Activity within the BRS at the level of the body directly influences neural networks and neural networks affect functioning of the BRS at the level of the body. Neural networks in the brain give rise to both conscious and nonconscious levels of processing. Of particular importance to models/ theories of behavior change is that, for the most part, they operate at the level of conscious processing and ignore the fact that neural networks below the level of consciousness are critically important to health behavior and health behavior change. We also want to emphasize that BRS of the body can effect behavior through both conscious and nonconscious processing. Because our relational experience alters biological regulatory structures of the body and brain, these experiences also affect health behavior through these same pathways. This is readily apparent in how social and physical environmental factors support obesogenic behavior, including physical inactivity. ${ }^{35}$

\section{The embodied mind}

In addition to addiction, there is a large body of literature supporting the notion that biological regulatory systems influence health behavior either through their effects on conscious subjective experience or via nonconscious processes. An example of such nonconscious effects that comes to mind is the phenomenon termed "sickness behavior", a cluster of behaviors including decreased movement and increased time spent sleeping, lack of appetite, and the propensity for social isolation. Specifically, what we now know is that the release of interleukin-1 from the immune system stimulates the vagus nerve and, independent of the specific illness, has effects on the central nervous system that fuel this cluster of behavior. ${ }^{36}$ Perhaps an even more glaring reminder of the embodied mind is depression. Tiermeier, ${ }^{37}$ underscoring the public health significance of this disease in late life, concluded that over $50 \%$ of those with severe depression have disturbed glucocorticoid feedback mechanisms. Depression is also common with increasing comorbid conditions associated with aging, a phenomenon that appears to be related to inflammation and cell-mediated immune activation. ${ }^{38}$ Not surprisingly, researchers have investigated the adverse effects that depression has on expectations and motives to engage in desired health behavior. For example, it is well known that depression is related to obesity ${ }^{39}$ and sedentary behavior. ${ }^{40}$ Additionally, there is evidence that digestive

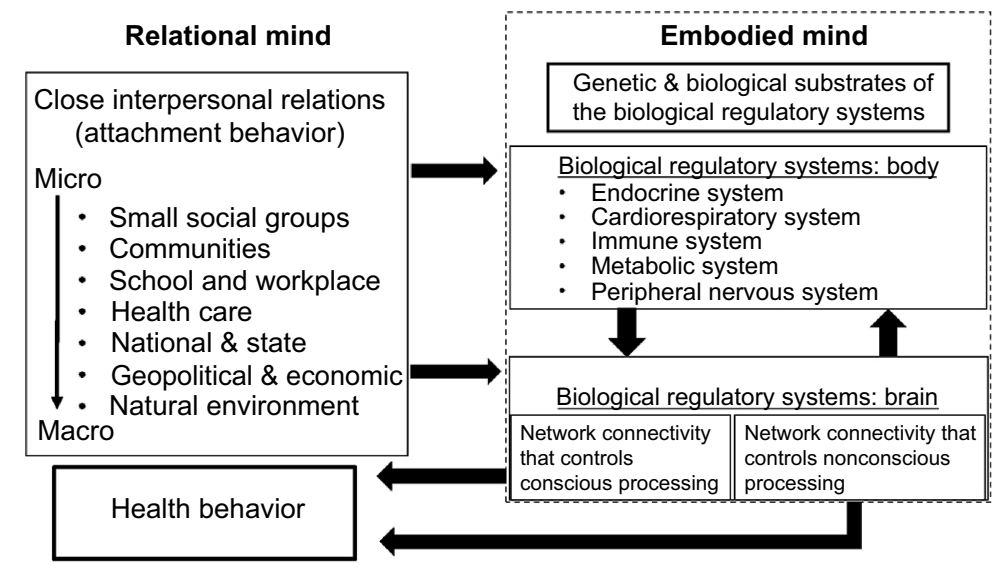

Figure 4 An embodied and relational model of health behavior. 
health plays a role in affect and emotion ${ }^{41}$ and that gut bacteria can motivate people to pursue the consumption of specific macronutrients. ${ }^{42}$ Data suggest that inflammation is a correlate of inactivity. ${ }^{43-45}$ Moreover, body fat is associated with increased inflammation, whereas intentional weight loss in older adults lowers body fat and reduces inflammation. ${ }^{46}$

Equally important is an awareness and appreciation of the fact that dynamic changes in biological regulatory systems and their substrates across relatively brief periods can profoundly influence functional brain networks and subjective states. For example, in a study of obese older adults, we found that craving for favorite foods dramatically increased over a period of $3 \mathrm{hrs}$ when restricted to consume water only versus ingesting a meal replacement. ${ }^{47}$ Even more interesting was the fact that following this brief fasting period, functional imaging of the resting state brains in the water only condition looked similar to what you would see in other addictive behaviors, brain states that differed dramatically from resting states taken following consumption of a meal replacement.

We do not want to create the impression that we are encouraging investigators to treat subjective experience as subordinate to objective biological influence in the study of health behavior. In this regard, we want to make two points. First, we believe that biological regulatory systems play a particularly potent role in certain health behaviors. This point has been made for addiction. ${ }^{33}$ Yet, even in the case of addiction, it is clear that the precise embodiment of micro-social environments is not a given. "Medical researchers are correct that the brain changes with addiction. Nevertheless, the way it changes has to do with learning and development-not disease. Addiction can therefore be seen as a developmental cascade, often foreshadowed by difficulties in childhood" (page xiii). ${ }^{2}$

Second, there is evidence that the role of biology in health behavior likely interacts with a person's subjective sense of agency. For example, in a prospective study, we tracked 480 older men and women who had knee pain on most days of the week to examine how lower leg strength and baseline self-efficacy influence decline in stair climbing performance across 30 months. ${ }^{48}$ What we observed was that older adults with low strength (estimated at the 25th percentile) and low self-efficacy (estimated at the 25th percentile) experienced a 4.15-s decrease in their stair climb performance that was statistically inferior in performance to any other subgroup. The other subgroups (high strength and low self-efficacy; low strength and high self- efficacy or high strength and low self-efficacy) lost about $1.30 \mathrm{~s}$ in their stair climb time and were not distinguishable from one another. In short, having high self-efficacy for the stair climb task buffered the effects that low strength was expected to have on decline in task performance.

Indeed, self-efficacy nicely illustrates the interplay between bodily states and the brain. The extent to which one's self-efficacy beliefs are under the influence of fluctuations in biology differs depending on an individual's experience in the behavior at hand. For a novice, efficacy beliefs are volatile and likely to change in response to shifting biological and psychosocial states, for example, momentary increases in muscle soreness and fatigue. Conversely, those with experience tend to display stability in their beliefs regarding their capability, and these beliefs are likely to persist despite momentary setbacks. ${ }^{49}$

Whereas dynamic biological systems can influence the motivation to approach or desire specific substances or outcomes, equally important is how the body influences the motivation to avoid behavior. In fact, Porges ${ }^{50}$ has argued that a major evolutional attribute of the human nervous system is the nonconscious motive to identify threat and thus to avoid harm to oneself. In fact, this probably explains why, when threatened even modestly as is true with many health events, people have a hard time thinking about anything but escaping the threat. Within the nervous system, the ventral root of the vagus nerve serves this function and one can estimate activity of this pathway linking body to brain via a biomarker known as respiratory sinus arrhythmia (RSA). ${ }^{50}$ As a person is threatened and the threat exceeds resources, RSA decreases and there is a concomitant increase in sympathetic nervous system activity. A major consequence of a decrease in RSA is that the brain becomes less reflective and acts automatically as a means of neutralizing the threatfavoring neural pathways that result in a rapid response. This distinction between the capacities for slow, reflective cognitive processing of input versus a fast, automatic mode is a core principle of dual-processing models of social cognition. ${ }^{51,52}$ Both low tonic levels of RSA (resting) and high phasic levels in response to withdrawal from stress are relevant to behavior change because they can promote behavioral and affective responses that can be inconsistent with consciously stated goals: "I know that I should stop eating snack food, but it helps me to get through the strain in my marriage." Potential consequences of a dysfunctional vagal brake in aging might include outcomes such as a decrease in life space, social isolation, a lack of openness to adopting 
preventive health behaviors, increased likelihood of relapse, excessive sedentary behavior, dropping out of treatment, and the exacerbation of pain.

We want to end this section by pointing out that biological regulatory systems are likely to be useful in understanding individual differences in response to behavioral interventions. For example, Hendershot and colleagues ${ }^{11}$ reviewed the growing literature of genetic influences on treatment response and relapse. They concluded that genetic polymorphisms moderate treatment effects for a variety of addictive behaviors including smoking, alcohol, and drug abuse through a range of metabolic and neurotransmitter pathways. Recently, research from our $1 \mathrm{ab}{ }^{53}$ used baseline dynamic brain networks from functional magnetic resonance imaging (fMRI) to identify older, obese, adults most likely to succeed in a behavioral weight loss intervention. We combined machine learning and functional brain networks to produce multivariate prediction models using baseline data to predict success with weight loss (a median split on percent weight lost) following 18 months of treatment. Older adults above the median lost on average $13.96 \%$ of the body weight, whereas it was $2.87 \%$ for those below the median. The prediction accuracy of our model was $95 \%$ as compared to static and random models that were either at or below $50 \%$. Principal component analysis of the data suggested that effective self-regulation involved both nonconscious and conscious processes. ${ }^{53}$

\section{The relational mind: attachment}

Having established the concept of the mind as an embodied process, we next examine why it is also important to consider the interpersonal, relational nature of the mind as an integral topic of theoretical inquiry into health behavior and aging. As infants come into the world, they directly connect with sources of energy and information flow communicated by significant others through physical proximity, nonverbal cues, and vocal tones. Cozolino ${ }^{54}$ argues that just as neural synapses enable the flow of energy and information between neurons, people also exchange the flow of energy and information with others via social synapses. Shaw and colleagues, using a large national database, ${ }^{55}$ found that a lack of emotional support from parents early in life was prospectively related to increased depression and chronic health conditions that persisted across the lifespan. Moreover, Mate ${ }^{56}$ in a Canadian bestseller argued persuasively that early emotional disturbances steer people toward addiction. He is not alone in this promoting this thesis, and it would appear relevant to a range of health behavior including drugs, alcohol, smoking and even dietary choices. ${ }^{57}$

Polyvagal theory provides a strong theoretical rationale for the fact that it is the ventral vagus nerve and its network of connectivity with other cranial nerves in the brainstem that serves as the main neural enabler of social connection in infancy and throughout the lifespan. Positive attachment, via this embodied network of connection with others, results in feelings of safety and security. Logically, polyvagal theory ${ }^{50}$ posits that safety established through social connection is the primary need state of humans (note the overlap with core needs within SDT) and that failure to satisfy this need results in a variety of psychiatric complications. In fact, there is a substantial body of literature linking developmental dysfunction in RSA to psychopathology. ${ }^{58}$ What we do not know at this point is whether developmental deficiencies in RSA lead to excessive health care utilization and accelerated rates of disability as people age.

\section{The relational mind: micro- and macro- social/physical environments.}

Because human relations extend well beyond primary caregivers, as discussed and depicted in Figure 4, health behavior is profoundly affected by micro- and macro-relational effects. The effects are often due to "...constraints that limit choice and the role of normative structures that shape the social values attached to activities, identities and choices. It also engages themes of inequality and power in society" (pp. 79-80). ${ }^{1}$ While one might typically conceive of these influences as exclusively social in nature, through experience, people become part of a relational fabric of existence with their physical and economic environments. For example, it is well known that physical features of neighborhoods, such as walkability ${ }^{59}$ and safety ${ }^{60}$ play a role in the physical activity behavior and social interactions of older populations.

Without question, a powerful social influence on health behavior of older adults, beyond early attachment, involves family members' role in scheduling screening visits, managing medications, and noticing changes in function that warrant medical attention. ${ }^{61}$ While these effects are generally favorable, family members can also have a negative impact on the health of older family members by being overprotective, and through various means restrict their life space and activity levels. These adverse consequences reflect the powerful role that ageism has on the health behavior of older adults. ${ }^{62}$ 
Interestingly, there is evidence that as the complexity and size of social networks decline with aging, the effect of social forces on health behavior also decreases. ${ }^{63}$ Perhaps this is one area where careful application of technology could be useful. Specifically, facilitating regular face-to-face communication (eg, via video telephony) with peers and with health care providers may be especially powerful on the well-being of individuals who are socially isolated. However, it is important that the use of technology is carefully considered, as increasing perceived isolation can be an adverse side-effect as well. For instance, the widespread use of automation in digital health interventions should be balanced with the cost and time burden of personal contacts, as automated programs may reduce meaningful face-to-face interactions with health care providers. In fact, there is emerging evidence that younger individuals who are the highest users of social media sites, which often maximize brief and effortless contacts instead of more effortful face-to-face communication, perceive greater isolation. ${ }^{64}$ Still, with a careful approach to the design of digital health tools, robust social contacts can be extended to individuals who are at present socially and geographically isolated.

Also deserving attention is the topic of multiple exposures to toxic micro- and macro-relational environments. Lynch and colleagues ${ }^{65}$ reported in the New England Journal of Medicine on how cumulative economic hardship affects the functional health of older adults. They defined economic hardship as an income $<200 \%$ of the poverty level in 1965, 1974, and/or 1985. They found a strong-graded association between the number of times individuals were classified experiencing economic hardship $(0,1,2$, or 3$)$ and the risk ratios for poor physical, cognitive, and psychological health. Others have reported a dose-response relationship between both the number and duration of toxic relational environments and disease risk. ${ }^{66,67}$ Glass and McAtee ${ }^{29}$ concluded that late life appears to be a period of increasing vulnerability to the cumulative effects of disadvantaged social and economic environments.

As aging research on health behavior advances, we want to emphasize our belief that researchers should pay more attention to micro-relational effects on health behavior, specifically, a focus on physician-patient interactions and the value of small group interactions on the health behavior of older adults. We base this position on the knowledge that attachment behavior is a lifespan need with peaks at both ends of the age spectrum. As espoused by polyvagal theory, ${ }^{50}$ self-determination theory, ${ }^{16}$ and advances in neuroscience, ${ }^{34}$ we are wired for and need close human relationships. Epstein ${ }^{68}$ has called upon medicine to rebuild a health care system that prioritizes attentive listening and compassion, a shift that would benefit the health of physicians and provide meaningful, close interpersonal experiences for patients. Illness, disease and the loss of function that occur with aging are major sources of threat to personal safety. In the midst of these threats, a powerful antidote can be active listening and compassion in the care of older adults.

Likewise, we believe there is opportunity in leveraging the social power of small group interactions between older adults. As an example, we have been promoting groupmediated behavioral interventions as a means of delivering movement-based and weight loss programs to older adults for close to 20 years. $^{69-72}$ Run in small groups of $8-15$ participants, they use the group as an agent of change, that is, as a vehicle to promote self-regulatory skills, to enhance a sense of agency, and to increase commitment to change. Consistent with Epstein, ${ }^{68}$ group leaders are taught to model and promote active listening and compassion among group members. Moreover, the group is used as a means of bringing awareness to what $\mathrm{Neff}^{73}$ terms "community humanity", that is, an appreciation for the fact that you are never alone in the struggle to make change or to deal with behavior change in the face of adversity.

In closing this section, one point we want to emphasize is that as one moves from micro- to macro-relational effects, level of personal control decreases. For example, there is no doubt that social programs and health policies influence the health behavior of older adults; however, the average individual has no control over how these programs or policies operate. Clearly, however, as we have observed with federal laws related to smoking, macro-level influences on health behavior warrant serious attention due to their potential effect on population health.

\section{Summary and conclusions}

There are several areas identified in this review that are important to aging research on health behavior. First, health behavior is highly influenced by dynamic in-the-moment processes that may originate in the environment, the body, and the brain ${ }^{11,74}$ suggesting that the concept of awareness should be key to theories of behavior change. Awareness is a multidimensional concept, including in-the-moment awareness of (a) the target behavior, (b) the processes that lead to the behavior, and (c) action plans to interrupt these in-the-moment processes. To 
this end, we believe that emerging digital health tools, such as in-the-moment self-reports enabled via ecological momentary assessment and connected monitoring devices (eg, activity monitors, location sensors) offer tremendous resources for enhancing awareness of behaviors and factors that enhance or impede health behavior change. However, as with the application of technology for reducing social isolation, we would emphasize that digital health tools should be used in a manner that enhances awareness of one's behavior without fostering negative psychosocial states that act against an individual's ability to self-regulate. For instance, we would caution against delivering content on a social media platform alongside unregulated content that increases stress and results in ruminative thought processes. Clearly, additional research is needed in the area of aging and the role of technology in health behavior change.

Additionally, the dynamic nature of health behavior casts doubt on the ability to adequately assess constructs using static study designs in which measures are taken at baseline, usually at a single time point during the day, and then again at one or two follow-up visits across the span of several weeks, months, or years. As we have shown, subjective states related to the regulation of health behavior can change in a matter of hours as a result of fluctuations in physiologic state. ${ }^{47}$ This lack of attention to withinperson variability compromises the goal of social science to understand mechanisms of health behavior change. This seems particularly important to the study of older adults given the variability created by aging biological systems, chronic health conditions, and the prevalence of physical symptoms such as pain and fatigue. This also holds implications for the design of health promotion interventions, which typically reply on a small number of weekly contacts between participants and their group members. This leaves individuals alone in their effort to overcome these powerful transient behavioral influences. Here again, mHealth tools may provide a bridge to social connection and to in-the-moment interventions during particularly challenging times.

Second, most theories of health behavior focus on conscious psychological processes, yet it is apparent that health behavior is highly influenced by nonconscious processes. ${ }^{74}$ Indeed, health behavior is often under the control of stimuli from the environment and signals within the body - automatic responses resulting from brain networks that have been shaped through experience dependent learning across the lifespan. ${ }^{75}$ Additionally, the digital age and advanced marketing strategies have accelerated the degree to which human behavior is subject to and controlled by environmental influence. With the nonconscious flow of energy and information empowered by fixated attention to internet-connected devices, powerful developmental experience, and rumination over multiple threats to the self, future theories and interventions on health behavior require expansion beyond conscious-derived constructs. In fact, one promising topic of influence in this area has been research on the role of implementation intentions in priming adaptive responses to contexts that normally shortcircuit attempts to change behavior. ${ }^{74}$

Third, it is clear that the relational nature of the human brain to the environment and to other people is central to health behaviors and attempts to change these behaviors. This was apparent in our discussion of the role that nonconscious processes play in health behavior. As noted previously, early life adversity created by impoverished social environments create "...constraints that limit choice and the role of normative structures that shape the social values attached to activities, identities and choices" (pp. 79-80). ${ }^{1}$ While this adversity may stem from poverty and limited resources, the lack of trust and connection to others caused by developmental trauma constitutes an underappreciated influence on health behavior. As noted by Duffy and colleagues, ${ }^{75}$ the negative impact of living in impoverished social environments and the existence of early attachment-related trauma on health behaviors are likely due to resultant dysfunction in brain networks that are critical to effective self-regulation as people age. The challenge these barriers create for behavior change are monumental and largely nonconscious.

Finally, in an attempt to understand and change health behavior, recent research suggests that greater attention should be devoted to the incentive value of health behaviors and affective processes that occur prior to, during and following the enactment of these behaviors. ${ }^{17}$ How can we design behavioral interventions to optimize affective processes that facilitate the adoption and maintenance of positive health behaviors among older adults? As reviewed in this paper, answers to this question likely require intervention development targeting multiple levels of influence. It also requires paying close attention to the psychophysical state of older adults. Life transitions such as the onset or exacerbation of chronic disease, the death of a spouse, and coping with the biology of aging can drain the incentive value from either adopting new health behaviors or maintaining good health practices in the midst of emotional discord. 


\section{Acknowledgments}

This paper was prepared for the keynote presentation at an NIA sponsored workshop for the Research Centers Collaborative Network (RCCN, December 2018) entitled "Achieving and Sustaining Behavior Change to Benefit Older Adults". Partial support for this paper was provided through NIH/NIA funding: R56 AG051624.

\section{Disclosure}

The authors declare that there are no conflicts of interest in this work.

\section{References}

1. Short SE, Mollborn S. Social determinants and health behaviors: conceptual frames and empirical advances. Curr Opin Psychol. 2015;5:78-84. doi:10.1016/j.copsyc.2015.05.002

2. Lewis. The Biology of Desire. New York: Public Affairs; 2015.

3. Rosenstock IM. Historical origins of health belief model. Health Educ Quart. 1974;2(4):328-335.

4. Janz NK, Becker MH. The health belief model - a decade later. Health Ed Quart. 1984;11(1):1-47. doi:10.1177/109019818401100101

5. Bandura A. Social Foundations of Thought and Action: A Social Cognitive Theory. Englewood Cliffs: Prentice-Hall; 1986.

6. Bandura A. Health promotion by social cognitive means. Health Educ Behav. 2004;31(2):143-164

7. Clark N, Janevic M. Individual theories. In: Riekert KA, Okene JK, Pbert L, editors. Handbook of Health Behavior Change. Vol. 4th. New York (NY): Springer; 2014:3-26.

8. Ajzen I. Models of human social behavior and their application to health psychology. Psychol Health. 1998;13(4):735-740. doi:10.1080/ 08870449808407426

9. Ajzen I. The theory of planned behaviour: reactions and reflections. Psychol Health. 2011;26(9):1113-1127. doi:10.1080/ 08870446.2011 .613995

10. Marlatt GA, Donovan DM. Maintenance Strategies in the Treatment of Addictive Behaviors. 2nd ed. New York: Guilford Press; 2005.

11. Hendershot CS, Witkiewitz K, George WH, Marlatt GA. Relapse prevention for addictive behaviors. Subst Abuse Treat Prev Policy. 2011;6:17. doi:10.1186/1747-597X-6-17

12. Bien T. Mindfulness-based replase prevention for addicitve behaviors: a clinician's guideline. J Psychiatric Mental Health Nurs. 2011;18(7):e19-e20. doi:10.1111/j.1365-2850.2011.01761.x

13. Bowen S, Witkiewitz K, Clifasefi SL, et al. Relative efficacy of mindfulness-based relapse prevention, standard relapse prevention, and treatment as usual for substance use disorders: a randomized clinical trial. JAMA Psychiatry. 2014;71(5):547-556. doi:10.1001/ jamapsychiatry.2013.4546

14. Deci EL, Ryan RM. Intrinsic Motivation and Self-Determination in Human Behavior. New York: Plenum; 1985.

15. Teixeira PJ, Carraca EV, Markland D, Silva MN, Ryan RM. Exercise, physical activity, and self-determination theory: a systematic review. Int J Behav Nutr Phys Act. 2012;9:78. doi:10.1186/1479-5868-9-78

16. Ryan RM, Deci EL. Self-determination theory and the facilitation of intrinsic motivation, social development, and well-being. Amer Psychol. 2000;55(1):68-78. doi:10.1037/0003-066X.55.1.68

17. Williams D, Rhodes RE, Conner MT. Affective Determinants of Health Behavior. New York: Oxford University Press; 2018.

18. Berridge KC, Robinson TE, Aldridge JW. Dissecting components of reward: 'liking', 'wanting', and learning. Curr Opin Pharmacol. 2009;9 (1):65-73. doi:10.1016/j.coph.2008.12.014
19. Rhodes RE, Gray SM. Affect in the process of action control of health-protecting behaviors. In: Williams DM, RERMTC, editors, Affective Determinants of Health Behavior. New York (NY): Oxford University Press;2018:21-47.

20. Rhodes RE, Kates A. Can the affective response to exercise predict future motives and physical activity behavior? A systematic review of published evidence. Ann Behav Med. 2015;49(5):715-731. doi:10.1007/s12160-015-9704-5

21. Bronfenbrenner U. The Ecology of Human Development. Cambridge (MA): Harvard University Press; 1979.

22. Sallis JF, Cervero RB, Ascher W, Henderson KA, Kraft MK, Kerr J. An ecological approach to creating active living communities. Annu Rev Public Health. 2006;27:297-322. doi:10.1146/annurev. publhealth.27.021405.102100

23. Fitzgibbon ML, Kong L, Tussing-Humphreys L. Understanding population health from multi-level and community-based models. In: Riekert KA, Ockene JK, Pbert L, editors. The Handbook of Health Behavior Change. Vol. 4th. New York (NY): Springer; 2014:22-41.

24. Owen N, Sugiyama T, Eakin EE, Gardiner PA, Tremblay MS, Sallis JF. Adults' sedentary behavior determinants and interventions. Am J Prev Med. 2011;41(2):189-196. doi:10.1016/j.amepre.2011.05.013

25. Prochaska JO, Redding CA, Evers KE. The transtheoretical model and stages of change. In: Glanz K, Rimmer BK, Viswanath K, editors. Health Behavior and Health Education: Theory, Research \& Practice. 4th ed. San Francisco, CA: Jossey-Bass; 2008:97-117.

26. Marteau TM, Ogilvie D, Roland M, Suhrcke M, Kelly MP. Judging nudging: can nudging improve population health? Bmj-Brit Med J. 2011;342:d228-d228. doi:10.1136/bmj.d228

27. Siegel DJ. The Mindful Brain. New York: W. W. Norton \& Co.; 2007.

28. Hebb DO. The Organizaion of Behavior. New York (NY): Wiley; 1949.

29. Glass TA, McAtee MJ. Behavioral science at the crossroads in public health: extending horizons, envisioning the future. Soc Sci Med. 2006;62(7):1650-1671. doi:10.1016/j.socscimed.2005.08.044

30. Lucas AR, Klepin HD, Porges SW, Rejeski WJ. Mindfulness-based movement: a polyvagal perspective. Integrative Cancer Therapies. 2018;17(1):5-15.

31. McEwen BS. From molecules to mind. Stress, individual differences, and the social environment. Ann N Y Acad Sci. 2001;935:42-49.

32. Dew B, Elifson K, Dozier M. Social and environmental factors and their influence on drug use vulnerability and resiliency in rural populations. J Rural Health. 2007;23:16-21. doi:10.1111/j.1748-0361.2007.00119.x

33. Nestler EJ, Aghajanian GK. Molecular and cellular basis of addiction. Science. 1997;278(5335):58-63.

34. Liebermann M. Social: Why are Brains are Wired to Connect. New York: Crown; 2014.

35. French SA, Story M, Jeffery RW. Environmental influences on eating and physical activity. Annu Rev Public Health. 2001;22:309-335. doi:10.1146/annurev.publhealth.22.1.309

36. Sternberg EM. The Balance Within: The Sceince Connecting Health and Emotions. New York: W.H. Freeman \& Co.; 2000.

37. Tiemeier H. Biological risk factors for late life depression. Eur J Epidemiol. 2003;18(8):745-750.

38. Maes M. Depression is an inflammatory disease, but cell-mediated immune activation is the key component of depression. Prog Neuropsychopharmacol Biol Psychiatry. 2011;35(3):664-675. doi:10.1016/j.pnpbp.2010.06.014

39. Blaine B. Does depression cause obesity?: A meta-analysis of longitudinal studies of depression and weight control. J Health Psychol. 2008;13(8):1190-1197. doi:10.1177/1359105308095977

40. Teychenne M, Ball K, Salmon J. Sedentary behavior and depression among adults: A review. Int $J$ Behav Med. 2010;17(4):246-254. doi:10.1007/s12529-010-9075-z

41. Mayer EA. Gut feelings: the emerging biology of gut-brain communication. Nat Rev Neurosci. 2011;12(8):453-466. doi:10.1038/ nrn3071 
42. Relman DA. The human microbiome and the future practice of medicine JAMA. 2015;314(11):1127-1128. doi:10.1001/jama.2015.10700

43. Nicklas BJ, Beavers DP, Mihalko SL, Miller GD, Loeser RF, Messier SP. Relationship of objectively-measured habitual physical activity to chronic inflammation and fatigue in middle-aged and older adults. JGMS. 2016;71(11):1437-1443.

44. Wirth K, Klenk J, Brefka S, et al. Biomarkers associated with sedentary behaviour in older adults: a systematic review. Ageing Res Rev. 2017;35:87-111. doi:10.1016/j.arr.2016.12.002

45. Nilsson A, Bergens O, Kadi F. Physical activity alters inflammation in older adults by different intensity levels. Med Sci Sports Exerc. 2018;50(7):1502-1507. doi:10.1249/MSS.0000000000001582

46. Beavers KM, Ambrosius WT, Nicklas BJ, Rejeski WJ. Independent and combined effects of physical activity and weight loss on inflammatory biomarkers in overweight and obese older adults. $J \mathrm{Am}$ Geriatr Soc. 2013;61(7):1089-1094. doi:10.1111/jgs.12321

47. Rejeski WJ, Burdette JH, Burns M, et al. Power of food moderates food craving, perceived control, and brain networks following a short-term post-absorptive state in older adults. Appetite. 2012;58:806-813. doi:10.1016/j.appet.2012.01.025

48. Rejeski WJ, Miller ME, Foy CG, Messier SP, Rapp S. Self-efficacy and the progression of functional limitations and self-reported disability in older adults with knee pain. JGSS. 2001;56B(5):S261-S265.

49. Tschannen-Moran M, Hoy AW. The differential antecedents of self-efficacy beliefs of novice and experienced teachers. Teach Teach Educ. 2007;23(6):944-956. doi:10.1016/j.tate.2006.05.003

50. Porges SW. The polyvagal perspective. Biol Psychol. 2007;74 (2):116-143. doi:10.1016/j.biopsycho.2006.06.009

51. Evans JS. Dual-processing accounts of reasoning, judgment, and social cognition. Annu Rev Psychol. 2008;59:255-278. doi:10.1146/ annurev.psych.59.103006.093629

52. Kahneman D. Thinking Fast and Slow. New York: Farrar, Straus and Giroux; 2011.

53. Mokhtari F, Rejeski WJ, Zhu Y, et al. Dynamic fMRI networks predict success in a behavioral weight loss program among older adults. Neuroimage. 2018;173:421-433. doi:10.1016/j.neuroimage.2018.02.025

54. Cozolino L. The Neuroscience of Human Relationships. New York: W.W. Norton \& Co.; 2010.

55. Shaw BA, Krause N, Chatters LM, Connell CM, Ingersoll-Dayton B. Emotional support from parents early in life, aging, and health. Psychol Aging. 2004;19(1):4-12. doi:10.1037/0882-7974.19.1.4

56. Mate G. In the Realm of Hungry Ghosts. Berkeley (CA): North Atlantic Books; 2010.

57. van der Kolk B. The Body Keeps Score: Brain, Mind, and Body in the Healing of Trauma. New York (NY): Penguin Books; 2014.

58. Beauchaine TP, Thayer JF. Heart rate variability as a transdiagnostic biomarker of psychopathology. Int J Psychophysiol. 2015;98(2 Pt 2):338-350. doi:10.1016/j.ijpsycho.2015.08.004

59. Towne SD Jr., Won J, Lee S, et al. Using walk score and neighborhood perceptions to assess walking among middle-aged and older adults. J Comm Health. 2016;41(5):977-988. doi:10.1007/s10900-016-0180-z

60. Won J, Lee C, Forjuoh SN, Ory MG. Neighborhood safety factors associated with older adults' health-related outcomes: A systematic literature review. Soc Sci Med. 2016;165:177-186. doi:10.1016/j. socscimed.2016.07.024

Clinical Interventions in Aging

\section{Publish your work in this journal}

Clinical Interventions in Aging is an international, peer-reviewed journal focusing on evidence-based reports on the value or lack thereof of treatments intended to prevent or delay the onset of maladaptive correlates of aging in human beings. This journal is indexed on PubMed Central, MedLine, CAS, Scopus and the Elsevier
61. Lee AA, Piette JD, Heisler M, Janevic MR, Langa KM, Rosland AM. Family members' experiences supporting adults with chronic illness: A national survey. Fam Syst Health. 2017;35(4):463-473. doi: $10.1037 /$ fsh0000293

62. Ory M, Hoffman MK, Hawkins M, Sanner B, Mockenhaupt R. Challenging aging stereotypes - Strategies for creating a more active society. AJPM. 2003;25(3):164-171.

63. Tucker JS, Klein DJ, Elliott MN. Social control of health behaviors: A comparison of young, middle-aged, and older adults. JGPS. 2004;59(4):P147-P150.

64. Primack BA, Shensa A, Sidani JE, et al. Social media use and perceived social isolation among young adults in the U.S. Am J Prev Med. 2017;53(1):1-8. doi:10.1016/j.amepre.2017.01.010

65. Lynch JW, Kaplan GA, Shema SJ. Cumulative impact of sustained economic hardship on physical, cognitive, psychological, and social functioning. NEJM. 1997;337(26):1889-1895. doi:10.1056/ NEJM199712253372606

66. House JS, Lepkowski JM, Kinney AM, Mero RP, Kessler RC, Herzog AR. The social stratification of aging and health. J Health Soc Behav. 1994;35(3):213-234.

67. Lantz PM, Lynch JW, House JS, et al. Socioeconomic disparities in health change in a longitudinal study of US adults: the role of health-risk behaviors. Soc Sci Med. 2001;53(1):29-40.

68. Epstein R. Attending: Medicine, Mindfulenss and Humanity. New York: Scribner; 2017.

69. Brawley LR, Rejeski WJ, Lutes L. A group-mediated cognitive-behavioral intervention for increasing adherence to physical activity in older adults. J Appl Biobehav Res. 2000;5(1):47-65. doi:10.1111/jabr.2000.5.issue-1

70. Rejeski WJ, Brawley LR, Ambrosius W, et al. Older adults with chronic disease: the benefits of group mediated counseling in the promotion of physically active lifestyles. Health Psychol. 2003;22 (4):414-423.

71. McDermott MM, Domanchuk K, Liu K, et al. The group oriented arterial leg study (GOALS) to improve walking performance in patients with peripheral arterial disease. Contemp Clin Trials. 2012;33(6):1311-1320. doi:10.1016/j.cct.2012.08.001

72. Rejeski WJ, Ambrosius WT, Burdette JH, Walkup MP, Marsh AP. Community weight loss to combat obesity and disability in at-risk older adults. JGMS. 2017;72(11):1547-1553.

73. Neff K. Self-compassion: an alternative conceptualization of a healthy attitude toward oneself. Self Identity. 2003;2:85-101. doi:10.1080/15298860309032

74. Sheeran P, Gollwitzer PM, Bargh JA. Nonconscious processes and health. Health Psychol. 2013;32(5):460-473. doi:10.1037/a0029203

75. Duffy KA, McLaughlin KA, Green PA. Early life adversity and health-risk behaviors: proposed psychological and neural mechanisms. Ann N Y Acad Sci. 2018;1428(1):151-169. doi:10.1111/ nyas. 13928

Bibliographic databases. The manuscript management system is completely online and includes a very quick and fair peer-review system, which is all easy to use. Visit http://www.dovepress.com/ testimonials.php to read real quotes from published authors. 